\title{
Interaction of Polyaniline with Surface of Carbon Steel
}

\author{
Maria P. Sokolova, ${ }^{1,2}$ Michael A. Smirnov, ${ }^{3,4}$ Igor A. Kasatkin, ${ }^{1}$ \\ Ivan Yu. Dmitriev, ${ }^{3}$ Natalia N. Saprykina, ${ }^{3}$ Alexander M. Toikka, ${ }^{1}$ \\ Erkki Lahderanta, ${ }^{2}$ and Galina K. Elyashevich ${ }^{3}$ \\ ${ }^{1}$ Saint Petersburg State University, Universitetskaya Nab. 7-9, Saint Petersburg 199034, Russia
${ }^{2}$ Lappeenranta University of Technology, Skinnarilankatu 34, 53850 Lappeenranta, Finland
${ }^{3}$ Institute of Macromolecular Compounds, Russian Academy of Sciences, Bolshoy Pr. 31, Saint Petersburg 199004, Russia
${ }^{4}$ ITMO University, Saint Petersburg 197101, Russia
}

Correspondence should be addressed to Maria P. Sokolova; pmarip@mail.ru

Received 22 January 2017; Revised 27 April 2017; Accepted 3 May 2017; Published 28 May 2017

Academic Editor: Andrea Camposeo

Copyright (C) 2017 Maria P. Sokolova et al. This is an open access article distributed under the Creative Commons Attribution License, which permits unrestricted use, distribution, and reproduction in any medium, provided the original work is properly cited.

\begin{abstract}
The structure and barrier properties of the oxyhydroxide layers on a carbon steel surface covered with electroactive polyaniline were investigated. Two types of polymer structure differing in degree of macromolecular order were prepared by simultaneous (fast polymerization) or dropwise (slow polymerization) mixing of reagents. A larger amount of the most stable FeOOH modification was formed on steel covered with slowly polymerized sample during treatment in the corrosion-active medium. Amorphous rust products with weak barrier properties were observed in the sample prepared by fast polymerization. Additionally, barrier activity of dedoped polyaniline was studied with SEM, WAXD, and electrochemical methods.
\end{abstract}

\section{Introduction}

Due to their unique physicochemical properties and easy synthesis, the electroconducting polymers attract considerable attention as potential components of electronic devices [1], power sources [2,3], chemical sensors [4], absorbents of electromagnetic radiation [5], and also corrosion protective coatings $[6,7]$. Their advantages include high electrical conductivity, low cost, and possibility of reducing environmental pollution $[8,9]$. Redox and catalytic activity of these materials promote formation of an oxyhydroxide layer on the polymer-metal interface [10-12], which passivates the metal surface and prevents it from corrosion; on the other hand, it causes instability of the electrical characteristics of the contact between the polymeric and metal phases [13]. Currently, the research in the field was mainly focused on the electrochemical investigations of the corrosion processes [14-18], but the oxide layer composition was not determined because of difficulties in applying ex situ experimental techniques $[19,20]$. For example, X-ray photoelectron spectroscopy (XPS) measurements $[9,11,21]$ are performed in vacuum where dehydration of the passive films takes place. Moreover, structure of the passive films is strongly affected by water content which changes dramatically under the conditions of XPS experiment [22, 23]. At the same time, X-ray diffraction (XRD) phase analysis can easily be performed at ambient conditions, which prevent dehydration of the passive film, and provides reliable information on its composition and crystalline structure [20]. Recently, this approach was used for investigation of polyaniline (PANI) ability to protect magnesium surface [24]. FTIR, Raman, and Mossbauer spectroscopy must be mentioned as an alternative for XRD method for studying structure of corrosion products at ambient conditions $[25,26]$. But in certain cases it is difficult to distinguish bands connected with inorganic phase on the background of spectrum of conducting polymer. The same problems can arise when using Raman spectroscopy. Additionally, irradiation of sample with laser beam can cause heating and changing of the structure of corrosion products (e.g., dehydration of iron oxyhydroxides). In comparison with Mossbauer spectroscopy, which can give quantitative information about phases of corrosion products of iron, XRD 
is more simple method. Additionally, the XRD patterns are well known for wide number of iron oxides and oxyhydroxides (including crystalline modifications, i.e., goethite, lepidocrocite, and akaganeite) and quantitive phase analysis of corrosion products is quite simple.

It needs to be pointed out that in some cases it is possible to use pure conducting polymer as a protective coating. It was proposed [27] that in this case conducting polymer acts as a catalyst for reduction of oxygen with iron, which results in formation of passive oxide layer on the surface of metal. Because of porosity and low mechanical stability of coating prepared with this simplest method, other approaches were elaborated: using of conducting polymer as a primer with conventional top-coat, blending with a conventional polymer coating, and addition of low concentration of conducting polymer to the conventional organic coatings [28]. It was shown that conducting polymer primer in combination with conventional top-coat demonstrates better performance than top-coat alone [29]. At the same time, blending of conducting polymer with conventional resins allows avoiding multilayer coating. It was reported that blended coatings formed by addition of equal amount of PANI or poly-o-ethoxyaniline to the water soluble epoxy or polymethylmethacrylate showed better corrosion protection of iron than single component coatings [30]. Additionally, it was shown that in some cases introduction of very small amount of conducting polymer can lead to the increasing of anticorrosion properties of conventional organic coatings. In work [31], it was shown that addition of less than 0.6 weight $\%$ of polyaniline to the alkyd resin leads to the improvement of corrosion resistance of the coating. Hybrid composite coating approach based on the simultaneous incorporation of inorganic (10 wt $\%$ of $\mathrm{ZnO})$ and PANI nanoparticles (2-5 wt \%) into poly(vinyl acetate) matrix was demonstrated in work [32] as a method for preparation of enhanced corrosion protective coatings.

Although various conducting polymers such as polypyrrole [33], polythiophene [34], and their derivatives [33, 35] are proposed as components of corrosion protection coatings, PANI and its derivatives are the most studied electroconducting polymers for corrosion protection [36] due to simplicity of their synthesis and high redox activity [37]. Polyaniline is a semicrystalline polymer with a typical degree of crystallinity $\left(\chi_{\mathrm{cr}}\right)$ of about 5-50\%; it can form at least two polymorphs [38-40]. Nanostructure and chain ordering in this polymer are strongly dependent on many synthesis parameters such as temperature, reagent concentrations, acidity of the reaction medium, and method of reagent mixing. PANI may be prepared in the form of nanoparticles with different shapes: nanospheres or granules [41-45], nanosheets [46, 47], nanofibers [48, 49], and nanotubes [50-52]. The mechanisms of PANI macromolecules ordering and nanostructure formation under different conditions are discussed in literature $[53,54]$. In a number of publications $[14,17,18,55]$, the effects of dopants and of the composition of PANI derivatives $[11,15,56-58]$ on the electrochemical properties of barrier layers formed under the deposition of polymer onto the metal surface were reported. At the same time, influence of polymer crystallinity and of its nanostructure on barrier properties was not studied in detail.
The aim of this work is to investigate chemical composition, crystalline structure, and barrier properties of inorganic films on the carbon steel/polyaniline interface formed with PANI prepared by two different procedures: dropwise mixing of reagents (slow polymerization) and simultaneous addition of an oxidant to the monomer solution (fast polymerization). The effect of dedoping of PANI on the products of its interaction with the steel surface is also discussed.

\section{Experimental}

2.1. Preparation of the Samples. Ammonium peroxydisulfate (APS) and aniline were purchased from Sigma-Aldrich and used as received. Sodium chloride and sulfuric acid were obtained from Vekton (Russia). Carbon steel (steel 08 GOST 1050-88) plates were polished with abrasive paper (P500 grit) and then washed with water and alcohol. All solutions were prepared in distilled water.

PANI dispersions were synthesized by chemical oxidative polymerization of aniline monomer $\left(0.15 \mathrm{~mol} \mathrm{~L}^{-1}\right)$ in the $\mathrm{H}_{2} \mathrm{SO}_{4}$ water solution $\left(1 \mathrm{~mol} \mathrm{~L}^{-1}\right)$ with APS as an oxidant. The mole ratio of monomer to oxidant was equal to $1: 1.125$. The monomer and oxidant solutions were thermostated separately at $35^{\circ} \mathrm{C}$ and then mixed together either simultaneously (sample P1) or dropwise (sample P2). After mixing, the solutions were left for 2 hours on the magnetic stirrer. Then the polymer was filtered and washed with the sulfuric acid solution in order to remove the residual ammonium sulfate. The polymer samples were dried in vacuum at room temperature during 48 hours. For dedoping of PANI, the powder of $\mathrm{P} 2$ was dispersed in $\mathrm{NH}_{4} \mathrm{OH}$ water solution with concentration of $1 \mathrm{~mol} \mathrm{~L}^{-1}$ and stirred for 1 hour. Then the solid polymer phase was separated by filtration, washed, and dried. The dedoped sample is denoted in text as P2d.

The polymer powders were dispersed in water under the ultrasonic treatment and casted onto metal surfaces (4-5 $\mathrm{mg} \mathrm{cm}^{-2}$ ) with subsequent drying on air. Then the samples were placed in the corrosion-active $3.5 \mathrm{wt} \% \mathrm{NaCl}$ solution in water. Those samples are denoted in text as "Fe$\mathrm{xxx}$," where " $\mathrm{xxx}$ " is $\mathrm{P} 1, \mathrm{P} 2$, or P2d.

\subsection{Characterization Methods}

2.2.1. Scanning Electron Microscopy. PANI powders were dispersed in water in the ultrasonic bath, casted on the metallic table in the same way as for the corrosion experiment, and studied with scanning electron microscope ZEISS MERLIN (Germany) at the voltage of $10 \mathrm{kV}$. Due to electroconductivity of PANI, the high quality images of the polymeric layers could be obtained without metal covering.

2.2.2. Wide-Angle X-Ray Diffraction. Initial PANI powders and oxide layers on the steel surface were investigated with wide-angle X-ray diffraction (WAXD) using a D2 PHASER diffractometer (Bruker, Germany) equipped with a $\mathrm{CoK}_{\alpha}$ radiation source $(\lambda=1.79 \AA)$ at scattering angles $2 \theta=$ $5^{\circ}-60^{\circ}$, step of $0.05^{\circ}$, and $2 \mathrm{~s}$ time per step. The XRD patterns were obtained immediately after deposition of PANI (prior to corrosion experiments) and after 8 days of treatment 


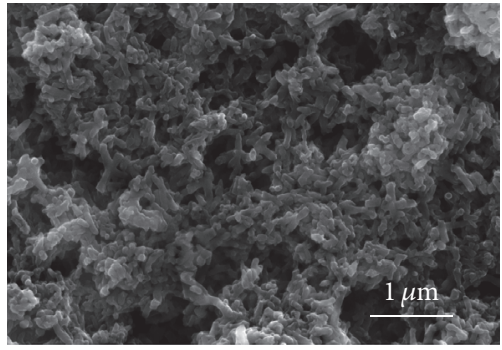

(a)

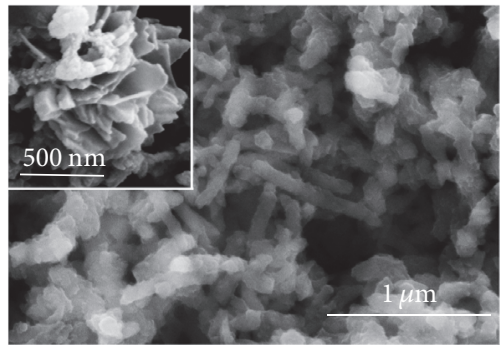

(d)

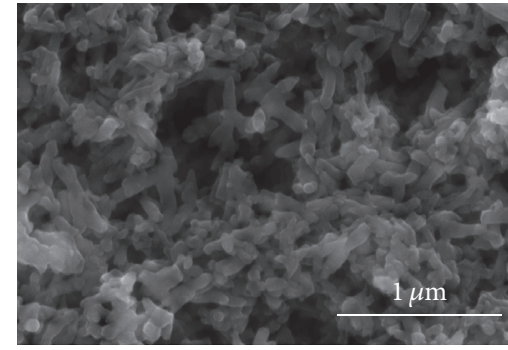

(b)

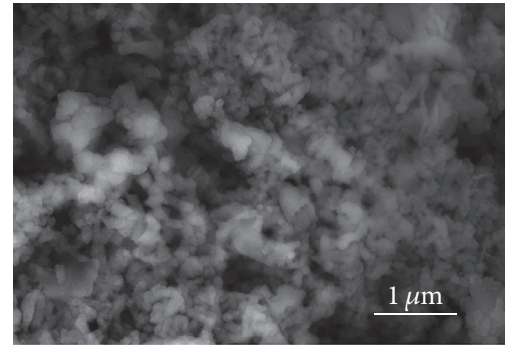

(e)

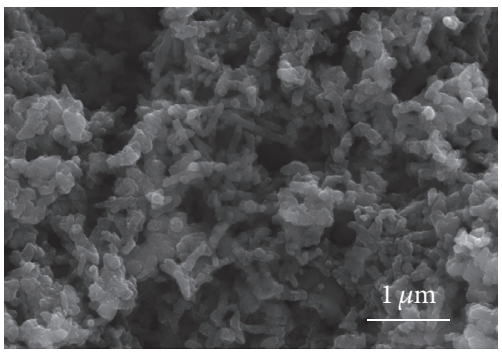

(c)

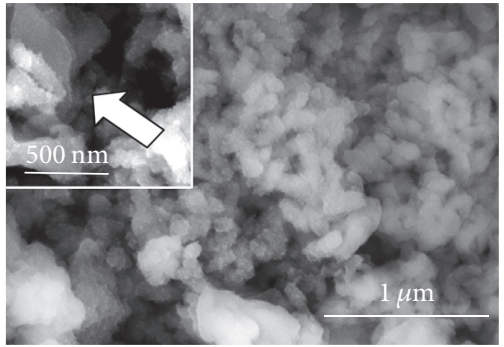

(f)

FIGURE 1: SEM images of PANI layers on metal surface. PANI prepared with simultaneous P1 (a, b) and dropwise P2 (c, d) addition of oxidant. Images (e) and (f) correspond to the dedoped polymer P2d.

in the corrosion-active medium. The Bruker TOPAS 4.2 software was used for quantitative phase analysis.

The degree of crystallinity of the samples $\left(\chi_{\mathrm{cr}}, \%\right)$ was calculated according to the equation:

$$
\chi_{\mathrm{cr}}=\frac{\int_{0}^{\infty} I_{\mathrm{cr}}(q) d q}{\int_{0}^{\infty}\left[I_{\mathrm{cr}}(q)+I_{\mathrm{am}}(q)\right] d q},
$$

where $I_{\mathrm{am}}$ and $I_{\mathrm{cr}}$ are diffraction intensities in the amorphous and crystalline regions, respectively, and $q$ is the length of the scattering vector.

2.2.3. Electrochemical Measurements. Barrier properties of the films were characterized by potentiodynamic experiment on the steel electrode with PANI coating using the P30-J potentiostat ("Elins," Russia). The experiments were carried out in a three-electrode cell with silver-chloride reference and Pt counter electrodes at 1st, 4th, and 8th days after immersion of the samples into the corrosion-active medium. The values of polarization resistance $\left(R_{p}\right)$ were calculated from the slope of the voltage-current dependence in the range of $\pm 20 \mathrm{mV}$ relative to the open-circuit electrochemical potential of the working electrode. All experiments were performed at room temperature.

\section{Results and Discussion}

3.1. Morphology of PANI Layers. Morphology of the polymer particles formed in the samples P1 and P2 (simultaneous and dropwise addition of the oxidant to the monomer solution, resp.) studied with scanning electron microscopy (SEM) is shown in Figure 1.

The sample P1 contains a large amount of smooth rodlike particles with the length in the range of $150-250 \mathrm{~nm}$ and diameter of about 50-100 nm (Figure 1(b)) which are uniformly distributed (Figure 1(a)) on the surface.

Dropwise addition of the oxidant to the monomer solution leads to the considerable changes in the polymer morphology. The SEM images of the sample P2 taken with a different magnification are shown in Figures 1(c) and $1(\mathrm{~d})$. As compared to the sample $\mathrm{P} 1$, the length of the rodlike particles increases to $200-1000 \mathrm{~nm}$, but the diameter remains approximately the same $(70-110 \mathrm{~nm})$. At the same time, the surface of the rods in $\mathrm{P} 2$ becomes rough that may be explained by the stepped character of the reaction during dropwise addition of the oxidant. It is seen in Figure 1(d) (insert) that the flake-like morphological elements are also present in the sample. Figure 1(c) shows that the P2 polymer layer contains larger amount of hollow space than the P1 layer (Figure 1(a)). The increased length of the particles may be related to the more effective polymer chain ordering in the slowly polymerized material.

Dedoping of PANI leads to the appearance of the particles with a less pronounced rod-like morphology as seen in Figure 1(e). The particles have a lower aspect ratio, and their sizes are in the range of $50-200 \mathrm{~nm}$. The flake-like morphological elements can be observed also in the P2d sample (marked with arrow in Figure 1(f)).

3.2. X-Ray Study of PANI Powders. The crystalline structure of the PANI samples was studied by WAXD. The results are presented in Figure 2. Wide diffraction peaks are observed for all samples and reveal their semicrystalline nature. WAXD pattern of the PANI powder prepared by simultaneous mixing of reagents (Figure 2(a)) shows two reflections at $2 \theta=$ $19.4^{\circ}$ and $25^{\circ}$, which correspond to the interplanar distances of $d=4.6$ and $3.5 \AA$, respectively. Similar results were obtained for the samples doped with $\mathrm{H}_{2} \mathrm{SO}_{4}$ by the authors 


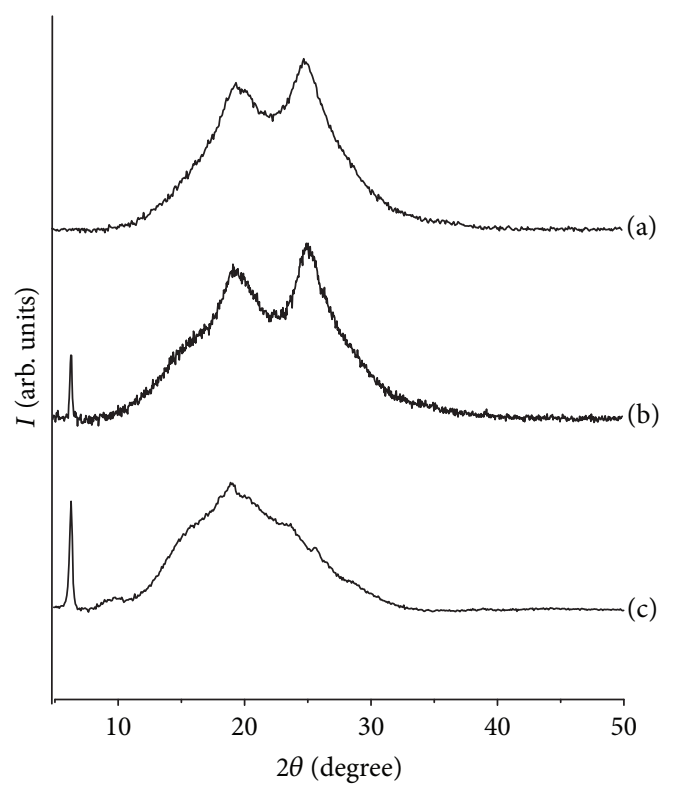

FIGURE 2: X-ray diffraction patterns of PANI powders prepared by simultaneous mixing of reagents, $\mathrm{P} 1$ (a), or dropwise addition of the oxidant, P2 (b), and by dedoping of P2 sample, P2d (c).

of $[44,49]$ who attributed the first peak to the characteristic distance between two adjacent chains of PANI and the second to the distance between the (110) planes.

When the method of mixing of reagents is changed to the dropwise addition of the oxidant (P2), the pattern of the polymer demonstrates an increased intensity of the peaks at $2 \theta=19.4^{\circ}$ and $25^{\circ}$ (Figure 2(b)), additional sharp peak at $2 \theta=6.4^{\circ}(d=13.9 \AA)$, and a shoulder with the maximum at about $2 \theta=15.5^{\circ}(d=5.7 \AA)$. This suggests an increased ordering of the polymer molecules that agrees with a higher degree of crystallinity of the P2 sample in comparison with P1 (31 and 23\%, resp.). In work [59], the sharp peak at $2 \theta=$ $6.4^{\circ}$ was attributed to the $2 \mathrm{D}$ stacking arrangement of the polymer chains with the dopant ions intercalated between the stacks. The authors of [52] suppose that the interplanar distance of $13.9 \AA$ could be attributed to the oligoanilines packed into the nanosheets with a lamellar arrangement of the molecules. Similar packing is observed in the case of large dopant anions which are able to form lamellar structures $[47,51,60]$. Presence of 2D-nanostructures is confirmed with SEM images shown in Figure 1(d).

The WAXD pattern of the dedoped sample P2d is shown in Figure 2(c). It is seen that the intensity of the reflection at $2 \theta=25^{\circ}$, which is attributed to the periodicity in the arrangement of dopant ions [44], is significantly reduced in comparison with the P2 sample. The degree of crystallinity also decreases after dedoping down to $9 \%$ that means amorphization of the polymer. At the same time, the peaks centered at $2 \theta=6.4^{\circ}$ and $19.4^{\circ}$ and the shoulder at $15.5^{\circ}$ remain at the same positions indicating that the molecular packing of the parent doped structure is preserved in some parts of the sample. It can be noted that the decrease in the intensity of the reflex at $2 \theta=25^{\circ}$ in the dedoped sample is associated with disappearance of the rod-like particles with high aspect ratio (Figure 1). Thus, the WAXD and SEM results evidence that the formation of particles with high aspect ratio is definitely related to the interaction between the PANI chains and the dopant ions.

\subsection{Chemical Composition of Barrier Inorganic Layers Prior} to and after Corrosion Experiments. Figure 3(a) shows the WAXD patterns of the PANI-covered samples taken prior to the corrosion experiment. The patterns obtained after keeping the samples in the $3.5 \% \mathrm{NaCl}$ solution during 8 days are shown in Figure 3(b). The peaks corresponding to the different phases are marked in the patterns. Crystallite sizes in the inorganic component calculated from the integral breadths of the diffraction peaks are listed in Table 1.

It was found that both of the doped PANI samples P1 and P2 (Figure 3(a), patterns (1) and (2)) start to react with the support immediately after the steel plate was covered by the polymer dispersion. The product of the reaction is the hydrous iron sulfate in two crystalline modifications: $\mathrm{FeSO}_{4} \times$ $7 \mathrm{H}_{2} \mathrm{O}$ (melanterite) and $\mathrm{FeSO}_{4} \times 4 \mathrm{H}_{2} \mathrm{O}$ (rozenite). This fact may be explained by the presence of an excess of dopant acid in the polymer layer that was determined by elemental analysis. The degree of PANI doping (the ratio between the number of acid molecules and nitrogen atoms in the polymer chain) was 0.90 and 0.74 for the P1 and P2 samples, respectively. The steel surface coated by the dedoped PANI layer remained lustrous after drying of polymer dispersion, and no inorganic phases were detected with XRD (Figure 3(a), pattern (3)). Thus, in contrast with the samples prepared with the doped polymers, the dedoped PANI does not immediately react with Fe during the deposition of polymer dispersion, due to the absence of dopant acid which is needed for providing counterions for the ferrous cations.

The phase composition of the inorganic layer after corrosion experiment appears to be different for all samples (Table 1). Not more than $5 \%$ of $\mathrm{NaCl}$ was detected in each sample (not shown in Table 1), which may be considered as an impurity coming from the corrosive solution used in our experiments. The broad peaks in the Fe-P1 WAXD pattern (Figure 3(b), pattern (1)) belong to $\alpha$-FeOOH (goethite) and $\mathrm{FeSO}_{4} \times 4 \mathrm{H}_{2} \mathrm{O}$ (rozenite). Goethite can appear as a product of iron oxidation reaction which is catalyzed by PANI according to the scheme suggested by Wessling [61]. A quantitative analysis of the Fe-P1 sample was not possible because of the low degree of crystallinity.

In the samples Fe-P2 and Fe-P2d, the sharp diffraction peaks are observed (Figure 3(b), patterns (2) and (3)). This means that relatively well-crystallized inorganic phases were formed under the coatings of PANI synthesized by dropwise addition of the oxidant. As it was mentioned above, the slow polymerization reaction allows formation of highly ordered rod-like and flake-like structures of the conducting polymer (Figure 2(d)). This could promote formation of more dense modification of iron oxyhydroxide and lead to an increase in the sizes of crystallites in the inorganic layers. As seen in Table 1, goethite is the dominating phase (81\%) in the sample $\mathrm{Fe}-\mathrm{P} 2$, while in the Fe-P2d the barrier layer is mostly formed by lepidocrocite (73\%). Goethite is the denser oxyhydroxide 


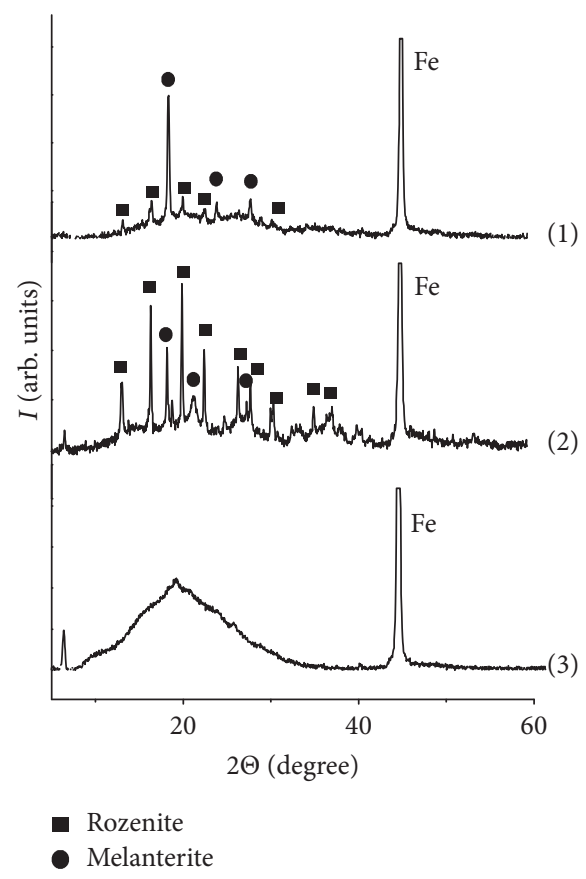

(a)

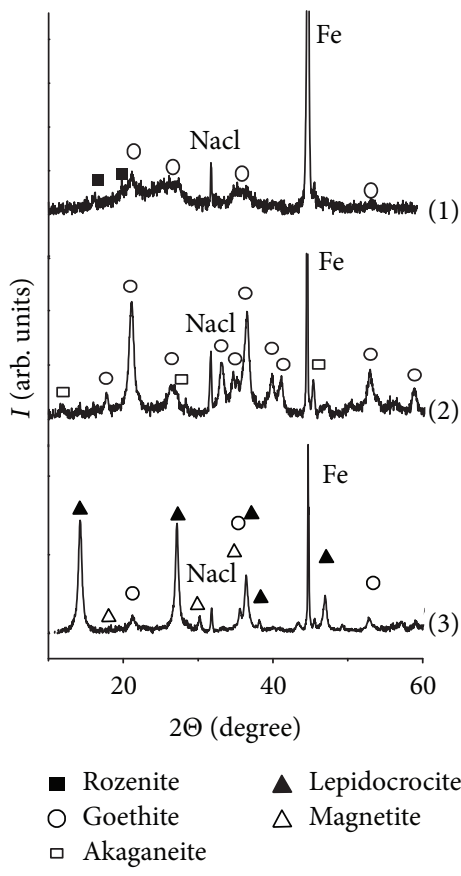

(b)

FIGURE 3: X-ray diffraction patterns of the samples Fe-P1 (1), Fe-P2 (2), and Fe-P2d (3) taken prior to (a) and after (b) corrosion experiments.

TABLE 1: Composition (in volume\%) and structural parameters ofinorganic layers at the PANI-Fe interface before and after corrosion experiment.

\begin{tabular}{lccc}
\hline Sample & Phase & Volume, $\%$ & Crystallite size, nm \\
\hline Fe-P1/before & Melanterite $\left(\mathrm{FeSO}_{4} \times 7 \mathrm{H}_{2} \mathrm{O}\right)$ & 85 & 34 \\
Rozenite $\left(\mathrm{FeSO}_{4} \times 4 \mathrm{H}_{2} \mathrm{O}\right)$ & 15 & 75 \\
\hline Fe-P1/after & Goethite $(\alpha-\mathrm{FeOOH})$ & - & - \\
& Rozenite $\left(\mathrm{FeSO}_{4} \times 4 \mathrm{H}_{2} \mathrm{O}\right)$ & - & 61 \\
Fe-P2/before & Rozenite $\left(\mathrm{FeSO}_{4} \times 4 \mathrm{H}_{2} \mathrm{O}\right)$ & 9 & 51 \\
\hline Fe-P2/after & Melanterite $\left(\mathrm{FeSO}_{4} \times 7 \mathrm{H}_{2} \mathrm{O}\right)$ & 81 & 13 \\
& Goethite $(\alpha-\mathrm{FeOOH})$ & 14 & 12 \\
\hline Fe-P2-b/before & Akaganeite $(\beta-\mathrm{FeOOH})$ & No inorganic phases detected & 13 \\
Fe-P2-b/after & Lepidocrocite $(\gamma$-FeOOH) & 73 & 11 \\
& Goethite $(\alpha-\mathrm{FeOOH})$ & 9 & 25 \\
\hline
\end{tabular}

phase in comparison with lepidocrocite. Taking into account the highest degree of crystallinity in the doped P2 sample, it may be supposed that the appearance of high amount of goethite is related to the high degree of ordering in the polymer chain packing.

3.4. Barrier Properties of Oxide Layers. Polarization curves of the steel electrodes with the PANI coatings were measured at the 1st, 4th, and 8th day of their keeping in the corrosionactive medium. The values of $R_{p}$ are presented in Table 2 . The polarization resistance of pure steel decreased from 37.1 to $22.5 \mathrm{kOhm}$ during the corrosion experiment. This may be explained by an increased surface roughness as a result of the electrode dissolution.
TABLE 2: Polarization resistance $R_{p}$ (in $\mathrm{kOhm}$ ) at different durations of corrosion experiment.

\begin{tabular}{lccc}
\hline Sample & $R_{p}$ at 1st day & $R_{p}$ at 4 th day & $R_{p}$ at 8 th day \\
\hline Fe & 37.1 & 24.4 & 22.5 \\
Fe-P1 & 7.9 & 18.1 & 29.9 \\
Fe-P2 & 12.4 & 16.4 & 83.3 \\
Fe-P2d & 1.4 & 41.4 & 67.8 \\
\hline
\end{tabular}

The $R_{p}$ values of all coated samples in the 1st day were lower than of pure steel. The $R_{p}$ value is inversely proportional to the sum of all currents of all electrochemical processes occurring on the electrode surface. In the initial 
period, the electrical contact between Fe support and PANI layer is good that leads to a fast oxidation of steel reflecting the catalytic activity of the conducting polymer in this process. It can be seen that the dedoped polymer is more active in iron oxidation because it demonstrates the lowest initial $R_{p}$ value in comparison with the Fe-P1 and Fe-P2 samples. This can be explained by two reasons. (1) As it was shown by WAXD, in the case of doped polymers, the layer of ferrous sulfate, which was formed even before the sample immersion into the corrosion-active medium, can increase the electrical resistance between the conducting polymer and the metal surface. (2) The doped polymers are characterized by a denser chain packing that leads to the slower diffusion of oxygen molecules and ions to the metal surface.

For the steel samples covered with PANI, the value of $R_{p}$ increases with time due to the formation of passive layers. On 8th day, the $R_{p}$ value for the $\mathrm{Fe}-\mathrm{P} 1$ sample is only slightly higher than that for the uncovered steel. This may be explained by the formation of amorphous inorganic oxide layers which provide only a weak corrosion protection. The samples Fe-P2 and Fe-P2d demonstrate the high values of $R_{p}$ (see Table 2) due to the crystalline oxide layers. The protection activity of Fe-P2 is higher than that of Fe-P2d that can probably be related to their structural features. The amount of goethite in the Fe-P2 sample is $81 \%$ while in Fe-P2d its concentration is only $14 \%$. At the same time, $\alpha$ - $\mathrm{FeOOH}$ has a maximum density (Table 1 ) and is the most thermodynamically stable polymorph $[62,63]$. Goethite is considered to be the most effective product for iron protection. Authors of $[64,65]$ have introduced the protective ability index of inorganic layers (PAI) which is calculated as the relation between the amounts of $\alpha-\mathrm{FeOOH}$ and other components of a barrier layer. In our case, the indexes are 5.8 and 0.17 for the samples Fe-P2 and Fe-P2d, respectively, which is in agreement with electrochemical measurements. At the same time, the stronger and weaker protection of steel covered with P2 and P2d, respectively, are associated with the size of inorganic crystallites: 13 and $19 \mathrm{~nm}$ for the main part of the inorganic phase in the Fe-P2 and Fe-P2d samples, respectively (see Table 1). It is in accordance with [62] where it was found that a decrease in crystallite size in oxyhydroxides leads to the improvement of their barrier properties.

The polarization curves measured after 8 days of exposure in the corrosion-active medium are shown in Figure 4. It can be seen that the curves for Fe-P2 and Fe-P2d are located below the ones for $\mathrm{Fe}$ and $\mathrm{Fe}-\mathrm{P} 1$ that agrees with the values of $R_{p}$ listed in Table 2. The slope of cathodic branches for the $\mathrm{Fe}-\mathrm{P} 2$ and Fe-P2d samples is higher compared to Fe and FeP1. This may be related to a possible reduction of the dense oxyhydroxide layer during polarization measurements.

\section{Conclusions}

Polyaniline dispersions were synthesized by two procedures: simultaneous (fast polymerization) and dropwise (slow polymerization) mixing of reagents. Lower polymerization rate permits to achieve a higher ordering in the arrangement of polymer chains, which leads to the increase in aspect ratio of the polymer particles. The coatings prepared from

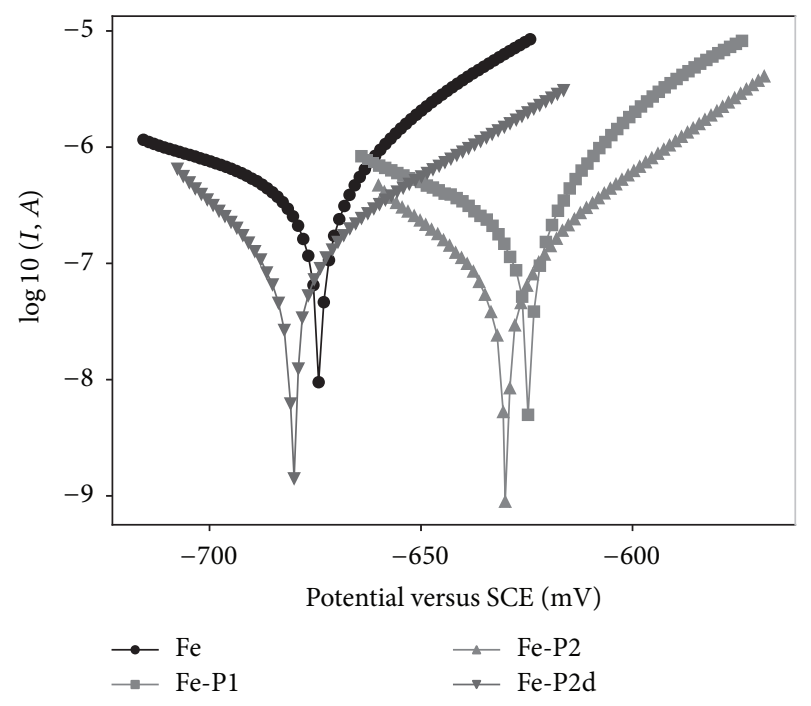

FIgURE 4: Polarization curves for pure Fe covered with PANI dispersions at 8 th day of keeping in $3.5 \% \mathrm{NaCl}$.

a polyaniline doped with sulfuric acid promote the formation of ferrous sulfate on the carbon steel surface. Different crystalline modifications of iron oxyhydroxides are formed during treatment of the samples in the corrosion-active solution (3.5\% NaCl in water). Deposition of a more ordered polymer onto the steel surface leads to the formation of a large amount (81\%) of the crystalline goethite phase which is the most thermodynamically stable rust product. Covering the steel with PANI prepared by common procedure (fast polymerization) leads to the formation of amorphous rust layer. For the inorganic layer formed under the polymer prepared by dropwise oxidant addition (slow polymerization), the electrochemical measurements show highest barrier properties which is confirmed by the data of structural investigations.

In a highly ordered dedoped PANI, the degree of the polymer crystallinity decreases from 31 to $9 \%$, and the rod-like structures disappear. Compared with the doped polyaniline, the dedoped sample displayed lower protective properties, which were still considerably higher than those of the doped PANI prepared by simultaneous mixing of reagents.

\section{Conflicts of Interest}

The authors declare that there are no conflicts of interest regarding the publication of this paper.

\section{Acknowledgments}

This work was supported by Russian Foundation for Basic Research (Grant 14-03-31411 mol_a). M. Sokolova acknowledges Saint Petersburg State University for the Research Grant (no. 12.50.1195.2014). The experimental work was facilitated by the equipment of the Center for X-Ray Diffraction Studies of Nanotechnology Interdisciplinary Center and Centre for Innovative Technologies of Composite Nanomaterials of Saint Petersburg State University. 


\section{References}

[1] B. Yaman, I. Terkesli, K. M. Turksoy, A. Sanyal, and S. Mutlu, "Fabrication of a planar water gated organic field effect transistor using a hydrophilic polythiophene for improved digital inverter performance," Organic Electronics: Physics, Materials, Applications, vol. 15, no. 3, pp. 646-653, 2014.

[2] M. A. Smirnov, M. P. Sokolova, N. V. Bobrova, I. A. Kasatkin, E. Lahderanta, and G. K. Elyashevich, "Capacitance properties and structure of electroconducting hydrogels based on copoly(aniline - P-phenylenediamine) and polyacrylamide," Journal of Power Sources, vol. 304, pp. 102-110, 2016.

[3] M. A. Smirnov, M. P. Sokolova, P. Geydt et al., "Dual doped electroactive hydrogelic fibrous mat with high areal capacitance," Materials Letters, vol. 199, pp. 192-195, 2017.

[4] H. J. N. P. D. Mello, T. Heimfarth, and M. Mulato, "Influence of the physical-chemical properties of polyaniline thin films on the final sensitivity of varied field effect sensors," Materials Chemistry and Physics, vol. 160, pp. 257-263, 2015.

[5] O. A. Andreeva, L. A. Burkova, M. A. Smirnov, and G. K. El'yashevich, "Correlation between IR spectra and electric conductivity of polyethylene-polypyrrole composites," Polymer Science-Series B, vol. 48, no. 11-12, pp. 331-334, 2006.

[6] B. N. Grgur, A. R. Elkais, M. M. Gvozdenović, S. Ž. Drmanić, T. L. Trišović, and B. Z. Jugović, "Corrosion of mild steel with composite polyaniline coatings using different formulations," Progress in Organic Coatings, vol. 79, pp. 17-24, 2015.

[7] Y. Li, H. Zhang, X. Wang, J. Li, and F. Wang, "Growth kinetics of oxide films at the polyaniline/mild steel interface," Corrosion Science, vol. 53, no. 12, pp. 4044-4049, 2011.

[8] G. K. Elyashevich, M. A. Smirnov, I. S. Kuryndin, and V. Bukošek, "Electroactive composite systems containing high conductive polymer layers on poly(ethylene) porous films," Polymers for Advanced Technologies, vol. 17, no. 9-10, pp. 700704, 2006.

[9] P. J. Kinlen, D. C. Silverman, and C. R. Jeffreys, "Corrosion protection using polyaniline coating formulations," Synthetic Metals, vol. 85, no. 1-3, pp. 1327-1332, 1997.

[10] P. Li, T. C. Tan, and J. Y. Lee, "Corrosion protection of mild steel by electroactive polyaniline coatings," Synthetic Metals, vol. 88, no. 3, pp. 237-242, 1997.

[11] A. A. Hermas, "XPS analysis of the passive film formed on austenitic stainless steel coated with conductive polymer," Corrosion Science, vol. 50, no. 9, pp. 2498-2505, 2008.

[12] Y. Li, H. Zhang, X. Wang, J. Li, and F. Wang, "Role of dissolved oxygen diffusion in coating defect protection by emeraldine base," Synthetic Metals, vol. 161, no. 21-22, pp. 2312-2317, 2011.

[13] J. Fang, K. Xu, L. Zhu, Z. Zhou, and H. Tang, "A study on mechanism of corrosion protection of polyaniline coating and its failure," Corrosion Science, vol. 49, no. 11, pp. 4232-4242, 2007.

[14] J. E. P. da Silva, S. I. C. de Torresi, and R. M. Torresi, "Polyaniline acrylic coatings for corrosion inhibition: the role played by counter-ions," Corrosion Science, vol. 47, no. 3, pp. 811-822, 2005.

[15] S. K. Shukla, M. A. Quraishi, and R. Prakash, "A self-doped conducting polymer 'polyanthranilic acid': an efficient corrosion inhibitor for mild steel in acidic solution," Corrosion Science, vol. 50, no. 10, pp. 2867-2872, 2008.

[16] J. R. Santos Jr., L. H. C. Mattoso, and A. J. Motheo, "Investigation of corrosion protection of steel by polyaniline films," Electrochimica Acta, vol. 43, no. 3-4, pp. 309-313, 1998.
[17] A. Cook, A. Gabriel, D. Siew, and N. Laycock, "Corrosion protection of low carbon steel with polyaniline: passivation or inhibition?" Current Applied Physics, vol. 4, pp. 133-136, 2004.

[18] A. R. Elkais, M. M. Gvozdenović, B. Z. Jugović, and B. N. Grgur, "The influence of thin benzoate-doped polyaniline coatings on corrosion protection of mild steel in different environments," Progress in Organic Coatings, vol. 76, no. 4, pp. 670-676, 2013.

[19] J. W. Schultze and M. M. Lohrengel, "Stability, reactivity and breakdown of passive films. Problems of recent and future research," Electrochimica Acta, vol. 45, no. 15-16, pp. 2499-2513, 2000.

[20] M. F. Toney, A. J. Davenport, L. J. Oblonsky, M. P. Ryan, and C. M. Vitus, "Atomic structure of the passive oxide film formed on iron," Physical Review Letters, vol. 79, no. 21, pp. 4282-4285, 1997.

[21] M. Fahlman, S. Jasty, and A. J. Epstein, "Corrosion protection of iron/steel by emeraldine base polyaniline: an X-ray photoelectron spectroscopy study," Synthetic Metals, vol. 85, no. 1-3, pp. 1323-1326, 1997.

[22] J. O. M. Bockris, "Spectroscopic observations on the nature of passivity," Corrosion Science, vol. 29, no. 2-3, pp. 291-312, 1989.

[23] J. Kruger, "The nature of the passive film on iron and ferrous alloys," Corrosion Science, vol. 29, no. 2-3, pp. 149-162, 1989.

[24] Y. Luo, Y. Sun, J. Lv, X. Wang, J. Li, and F. Wang, "Transition of interface oxide layer from porous $\mathrm{Mg}(\mathrm{OH})_{2}$ to dense $\mathrm{MgO}$ induced by polyaniline and corrosion resistance of $\mathrm{Mg}$ alloy therefrom," Applied Surface Science, vol. 328, pp. 247-254, 2015.

[25] J. A. Jaén, J. Iglesias, and C. Hernández, "Analysis of short-term steel corrosion products formed in tropical marine environments of Panama," International Journal of Corrosion, vol. 2012, Article ID 162729, 11 pages, 2012.

[26] A. V. R. Kumar and R. Balasubramaniam, "Corrosion product analysis of corrosion resistant ancient Indian iron," Corrosion Science, vol. 40, no. 7, pp. 1169-1178, 1998.

[27] B. Wessling and J. Posdorfer, "Corrosion prevention with an organic metal (polyaniline): corrosion test results," Electrochimica Acta, vol. 44, no. 12, pp. 2139-2147, 1999.

[28] P. P. Deshpande, N. G. Jadhav, V. J. Gelling, and D. Sazou, "Conducting polymers for corrosion protection: a review," Journal of Coatings Technology Research, vol. 11, no. 4, pp. 473494, 2014.

[29] A. J. Dominis, G. M. Spinks, and G. G. Wallace, "Comparison of polyaniline primers prepared with different dopants for corrosion protection of steel," Progress in Organic Coatings, vol. 48, no. 1, pp. 43-49, 2003.

[30] J. H. Huh, E. J. Oh, and J. H. Cho, "Investigation of corrosion protection of iron by polyaniline blend coatings," Synthetic Metals, vol. 137, no. 1-3, pp. 965-966, 2003.

[31] J. I. I. Laco, F. C. Villota, and F. L. Mestres, "Corrosion protection of carbon steel with thermoplastic coatings and alkyd resins containing polyaniline as conductive polymer," Progress in Organic Coatings, vol. 52, no. 2, pp. 151-160, 2005.

[32] R. C. Patil and S. Radhakrishnan, "Conducting polymer based hybrid nano-composites for enhanced corrosion protective coatings," Progress in Organic Coatings, vol. 57, no. 4, pp. 332336, 2006.

[33] D. E. Tallman, G. Spinks, A. Dominis, and G. G. Wallace, "Electroactive conducting polymers for corrosion control: part 1. General introduction and a review of non-ferrous metals," Journal of Solid State Electrochemistry, vol. 6, no. 2, pp. 73-84, 2002. 
[34] U. Riaz, C. Nwaoha, and S. M. Ashraf, "Recent advances in corrosion protective composite coatings based on conducting polymers and natural resource derived polymers," Progress in Organic Coatings, vol. 77, no. 4, pp. 743-756, 2014.

[35] E. Armelin, R. Oliver, F. Liesa, J. I. Iribarren, F. Estrany, and C. Alemán, "Marine paint fomulations: conducting polymers as anticorrosive additives," Progress in Organic Coatings, vol. 59, no. 1, pp. 46-52, 2007.

[36] P. Zarras and J. D. Stenger-Smith, "Electro-active polymer (EAP) coatings for corrosion protection of metals," in Handbook of Smart Coatings for Materials Protection, A. S. H. Makhlouf, Ed., pp. 328-369, Woodhead Publishing, 2014.

[37] G. G. Wallace, G. M. Spinks, L. A. P. Kane-Maguire, and P. R. Teasdale, Conductive Electroactive Polymers: Intelligent Polymer Systems, CRC Press, London, UK; Taylor \& Francis Group, New York, NY, USA, 2009.

[38] J. P. Pouget, M. E. Jozefowicz, A. J. Epstein, X. Tang, and A. G. MacDiarmid, "X-ray structure of polyaniline," Macromolecules, vol. 24, no. 3, pp. 779-789, 1991.

[39] M. E. Jozefowicz, R. Laversanne, H. H. S. Javadi et al., "Multiple lattice phases and polaron-lattice spinless-defect competition in polyaniline," Physical Review B, vol. 39, no. 17, pp. 12958-12961, 1989.

[40] M. E. Józefowicz, A. J. Epstein, J.-P. Pouget et al., "X-ray structure of polyanilines," Synthetic Metals, vol. 41, no. 1-2, pp. 723-726, 1991.

[41] H.-W. Park, T. Kim, J. Huh, M. Kang, J. E. Lee, and H. Yoon, "Anisotropic growth control of polyaniline nanostructures and their morphology-dependent electrochemical characteristics," ACS Nano, vol. 6, no. 9, pp. 7624-7633, 2012.

[42] E. A. Sanches, J. C. Soares, A. C. Mafud, G. Trovati, E. G. Fernandes, and Y. P. Mascarenhas, "Structural and morphological characterization of chloride salt of conducting poly(omethoxyaniline) obtained at different time synthesis," Journal of Molecular Structure, vol. 1039, pp. 167-173, 2013.

[43] J. Stejskal, M. Spirkova, A. Riede, M. Helmstedt, P. Mokreva, and J. Prokes, "Polyaniline dispersions 8 . The control of particle morphology," Polymer, vol. 40, no. 10, pp. 2487-2492, 1999.

[44] D. Zhu, J. Zhang, C. Xu, and M. Matsuo, "The frequencydependence conduction of polyaniline based on their paracrystalline structures," Synthetic Metals, vol. 161, no. 17-18, pp. 1820-1827, 2011.

[45] A. A. Ganash, F. M. Al-Nowaiser, S. A. Al-Thabaiti, and A. A. Hermas, "Protection of stainless steel by the electrodeposition of polyaniline/poly(o-phenylenediamine) composite layers," Journal of Solid State Electrochemistry, vol. 17, no. 3, pp. 849-860, 2013.

[46] Z. D. Zujovic, C. Laslau, G. A. Bowmaker et al., "Role of aniline oligomeric nanosheets in the formation of polyaniline nanotubes," Macromolecules, vol. 43, no. 2, pp. 662-670, 2010.

[47] T. Jana, J. Chatterjee, and A. K. Nandi, "Sulfonic acid doped thermoreversible polyaniline gels. 3-structural investigations," Langmuir, vol. 18, no. 15, pp. 5720-5727, 2002.

[48] E. A. Sanches, J. C. Soares, A. C. Mafud, E. G. R. Fernandes, F. L. Leite, and Y. P. Mascarenhas, "Structural characterization of Chloride Salt of conducting polyaniline obtained by XRD, SAXD, SAXS and SEM," Journal of Molecular Structure, vol. 1036, pp. 121-126, 2013.

[49] H. Zhang, X. Wang, J. Li, and F. Wang, "Facile synthesis of polyaniline nanofibers using pseudo-high dilution technique," Synthetic Metals, vol. 159, no. 14, pp. 1508-1511, 2009.
[50] C. Laslau, B. Ingham, Z. D. Zujovic et al., "Synchrotron Xray scattering reveals early-stage crystallinity during the selfassembly of polyaniline nanotubes with rectangular crosssections," Synthetic Metals, vol. 161, no. 23-24, pp. 2739-2742, 2012.

[51] L. J. Zhang, Y. Z. Long, Z. J. Chen, and M. X. Wan, “The effect of hydrogen bonding on self-assembled polyaniline nanostructures," Advanced Functional Materials, vol. 14, no. 7, pp. 693-698, 2004.

[52] J. K. Park, S. S. Jeon, and S. S. Im, "Effect of 4-sulfobenzoic acid monopotassium salt on oligoanilines for inducing polyaniline nanostructures," Polymer, vol. 51, no. 14, pp. 3023-3030, 2010.

[53] J. Stejskal, I. Sapurina, M. Trchová, E. N. Konyushenko, and P. Holler, "The genesis of polyaniline nanotubes," Polymer, vol. 47, no. 25, pp. 8253-8262, 2006.

[54] J. Stejskal, I. Sapurina, and M. Trchová, "Polyaniline nanostructures and the role of aniline oligomers in their formation," Progress in Polymer Science, vol. 35, no. 12, pp. 1420-1481, 2010.

[55] M. A. Smirnov, M. P. Sokolova, I. Y. Dmitriev, E. N. Vlasova, E. Y. Rozov, and G. K. El'yashevich, "Barrier properties and structure of inorganic layers at polyaniline-steel interface," Russian Journal of Applied Chemistry, vol. 88, no. 7, pp. 11681173, 2015.

[56] H. Bhandari, V. Choudhary, and S. K. Dhawan, "Influence of self-doped poly(aniline-co-4-amino-3-hydroxy-naphthalene1- sulfonic acid) on corrosion inhibition behaviour of iron in acidic medium," Synthetic Metals, vol. 161, no. 9-10, pp. 753-762, 2011.

[57] T. Mousavinejad, M. R. Bagherzadeh, E. Akbarinezhad, M. Ahmadi, and M. J.-F. Guinelac, "A novel water-based epoxy coating using self-doped polyaniline-clay synthesized under supercritical $\mathrm{CO}_{2}$ condition for the protection of carbon steel against corrosion," Progress in Organic Coatings, vol. 79, pp. 9097, 2015.

[58] M. P. Sokolova, N. V. Bobrova, I. Y. Dmitriev et al., "Anticorrosion activity of aniline-aniline-2-sulfonic acid copolymers on the steel surface," Russian Journal of Applied Chemistry, vol. 89, no. 3, pp. 432-438, 2016.

[59] R. Murugesan and E. Subramanian, "Effect of organic dopants on electrodeposition and characteristics of polyaniline under the varying influence of $\mathrm{H}_{2} \mathrm{SO}_{4}$ and $\mathrm{HClO}_{4}$ electrolyte media," Materials Chemistry and Physics, vol. 80, no. 3, pp. 731-739, 2003.

[60] L. Shi, X. Wu, L. Lu, X. Yang, and X. Wang, "Intercalated polyaniline nanosheets prepared from lyotropic liquid crystalline solutions and their capacitive performance," Synthetic Metals, vol. 160, no. 9-10, pp. 989-995, 2010.

[61] B. Wessling, "Passivation of metals by coating with polyaniline: corrosion potential shift and morphological changes," Advanced Materials, vol. 6, no. 3, pp. 226-228, 1994.

[62] J. Wei, X. X. Fu, J. H. Dong, and W. Ke, “Corrosion evolution of reinforcing steel in concrete under dry/wet cyclic conditions contaminated with chloride," Journal of Materials Science and Technology, vol. 28, no. 10, pp. 905-912, 2012.

[63] Y.-L. Zhou, J. Chen, and Z.-Y. Liu, "Corrosion behavior of rusted $550 \mathrm{MPa}$ grade offshore platform steel," Journal of Iron and Steel Research International, vol. 20, no. 3, pp. 66-73, 2013.

[64] T. Kamimura, S. Hara, H. Miyuki, M. Yamashita, and H. Uchida, "Composition and protective ability of rust layer formed on weathering steel exposed to various environments," Corrosion Science, vol. 48, no. 9, pp. 2799-2812, 2006. 
[65] F. R. Pérez, C. A. Barrero, O. Arnache, L. C. Sánchez, K. E. García, and A. R. Hight Walker, "Structural properties of iron phases formed on low alloy steels immersed in sodium chloriderich solutions," Physica B: Condensed Matter, vol. 404, no. 8-11, pp. 1347-1353, 2009. 

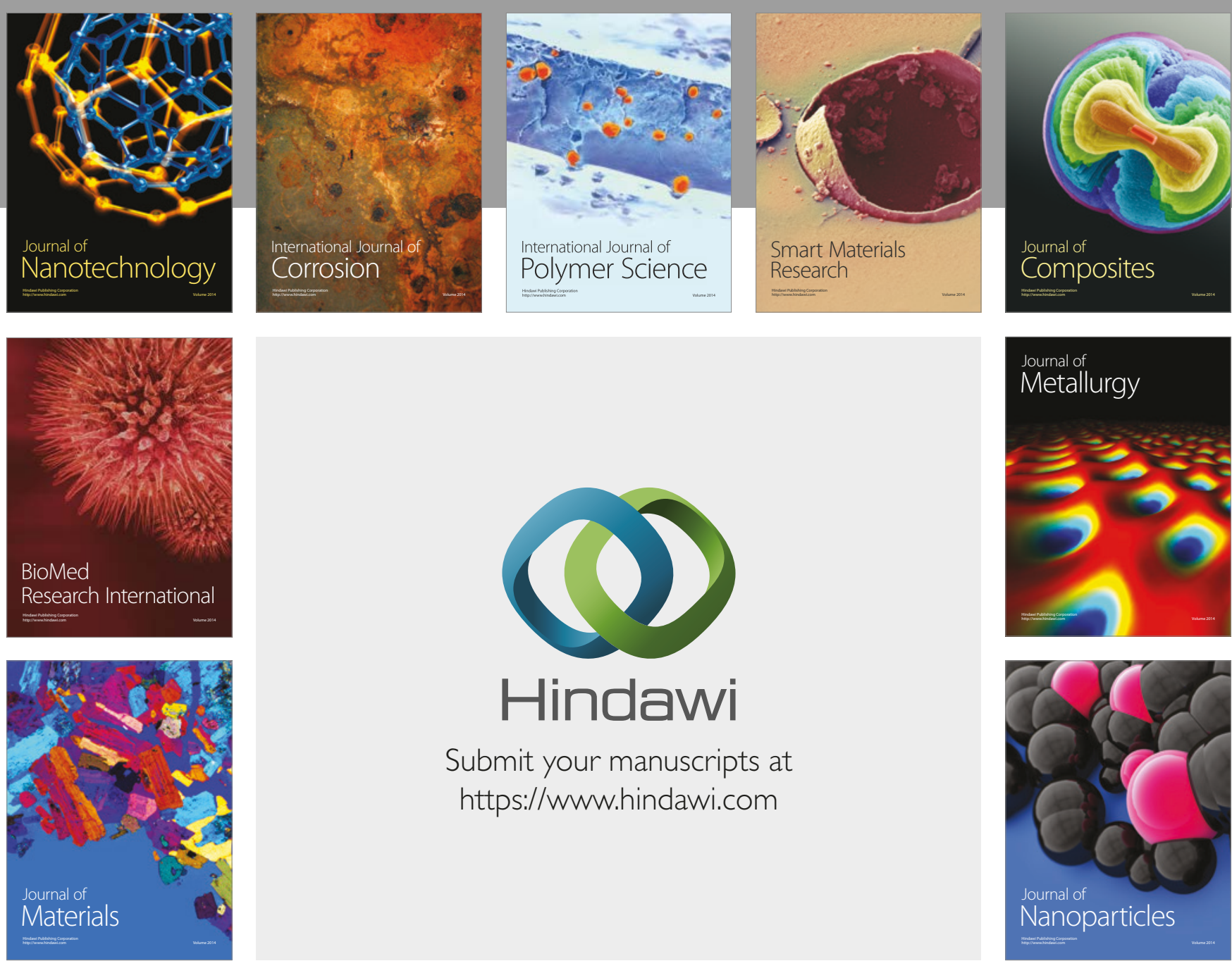

\section{Hindawi}

Submit your manuscripts at

https://www.hindawi.com
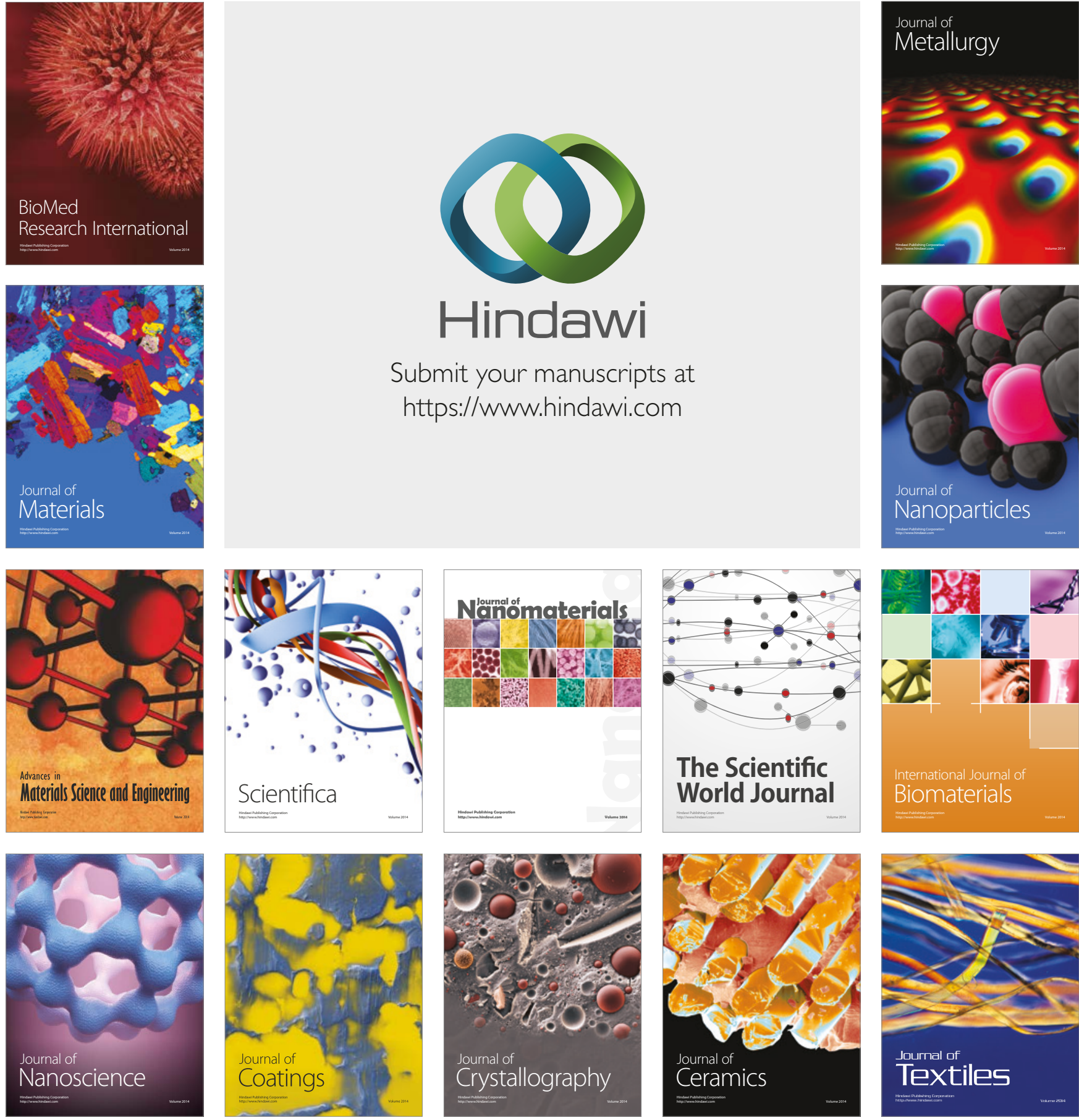

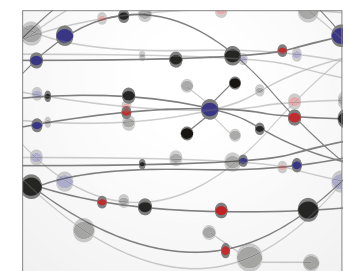

The Scientific World Journal
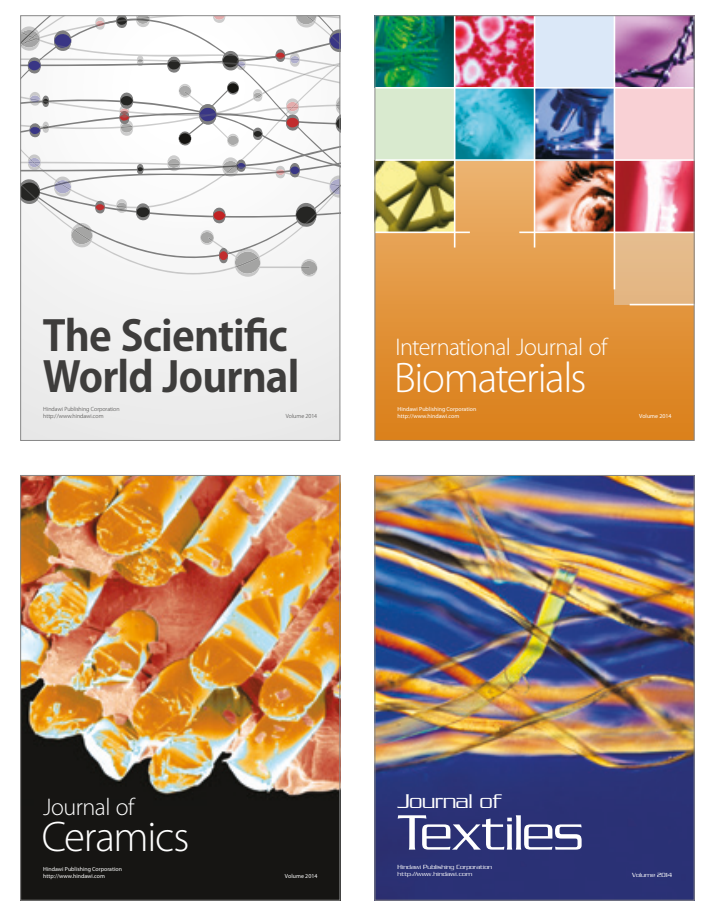